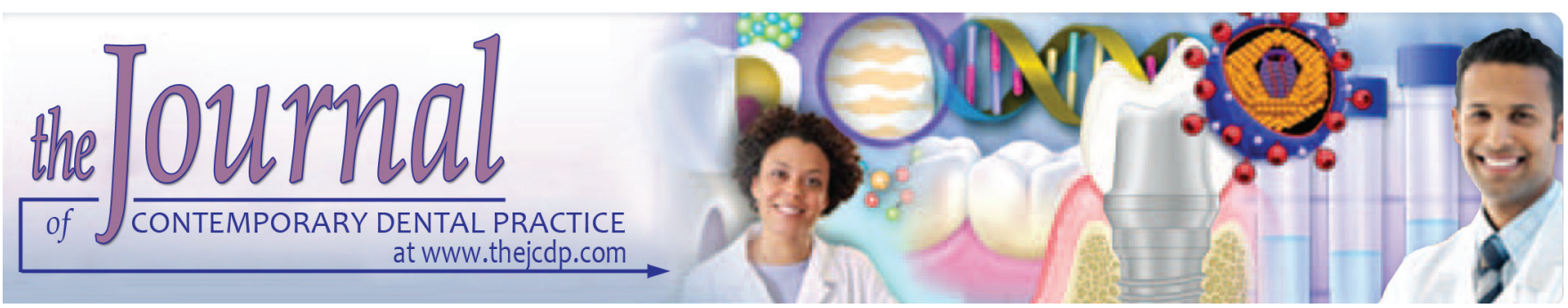

\title{
Veneer or Interproximal Enamel Reduction?
}

\section{Mohamed S Bamashmous}

\section{ABSTRACT}

Background: Clinicians often ask, "Veneer or no to veneer?" Clinicians usually think of porcelain veneers for cases in which patients are dissatisfied with their teeth shape. Interproximal reduction can change teeth's shape and appearance within the enamel to improve gingival contour, eliminate black gingival triangles, and correct the Curve of Spee.

Aim: Our aim is to present to clinicians the option of reshaping and interproximal reduction (IPR) as a valid alternative to porcelain veneers.

Case report: A male patient with round bulbous-shaped anterior maxillary teeth sought a better masculine smile. Interproximal reduction with thin bur size was performed to reshape the anterior teeth in order to achieve a proportional teeth width to height and give a more rectangular rather than square shape. Finally, the orthodontist closed the multiple diastemas using round wires in order to reduce anterior teeth proclination. The patient received bleaching treatment and minor composite fillings.

Conclusion: Not every cosmetic case should be treated with veneers and crowns. Conservative minimal intervention, such as IPR was more than sufficient in treating such cases. It is the right of the patients to be informed and educated about all possible treatment options.

Clinical significance: Minor reshaping and IPR preserving teeth structures can be helpful in achieving esthetic results and patients' confidence.

Keywords: Esthetics, Interproximal reduction, Shape, Veneers.

How to cite this article: Bamashmous MS. Veneer or Interproximal Enamel Reduction? J Contemp Dent Pract 2018;19(6):749-751.

Department of Dental Public Health, Faculty of Dentistry King Abdulaziz University, Jeddah, Kingdom of Saudi Arabia Department of Orthodontics, Henry M. Goldman School of Dental Medicine, Boston University, Boston, Massachusetts, USA

Corresponding Author: Mohamed S Bamashmous, Department of Dental Public Health, Faculty of Dentistry, King Abdulaziz University, Jeddah, Kingdom of Saudi Arabia; Department of Orthodontics, Henry M. Goldman School of Dental Medicine Boston University, Boston, Massachusetts, USA, e-mail: hamada_y2k@hotmail.com

\section{Source of support: Nil}

Conflict of interest: None

\section{BACKGROUND}

The ultimate goal of orthodontic treatment is to create functional occlusion, dental, and facial esthetics. Interproximal enamel reduction is an orthodontic treatment modality for creating or gaining space. ${ }^{1}$ The IPR is carried out by the reduction and anatomic recontouring of enamel structure. ${ }^{2}$ It also helps to adjust for Bolton Index discrepancy and sometimes, it is an alternative method to teeth extraction. The IPR can be used to change teeth's shape and dental appearance within the enamel surface, improve gingival contour, eliminate black gingival triangles, and correct the Curve of Spee. In the finishing stages of orthodontic treatment, it can enhance retention and treatment stability. ${ }^{3,4}$

There are several techniques for performing IPR using abrasive metal strips, diamond-coated stripping disks, and/or air-rotor stripping. ${ }^{2}$ In some cases, aggressive IPR can cause hypersensitivity, irreversible damage to dental pulp, increased plaque formation, caries risk, and periodontal diseases. Interproximal reduction should be performed only on patients with low caries risk and good oral hygiene. ${ }^{1,2}$

A study compared patients' reproximated mesiodistal enamel of anterior and posterior teeth after reduction and unground surfaces in the same patient. In their statistical analysis, they controlled for teeth brushing habits, use of dental floss and toothpicks, and regular fluoride supplementation. They found healthy dentitions with excellent occlusion and no significant difference in new caries lesions between reproximated $(2.5 \%)$ and unground $(2.4 \%)$ surfaces. None of the patients reported increased sensitivity to temperature variations. ${ }^{5,6}$ Our aim of the study is to present and educate clinicians about the option of reshaping and IPR as a valid alternative to porcelain veneers. 


\section{CASE REPORT}

A 26-year-old male patient had square-shaped, rounded corners anterior maxillary teeth (central and lateral incisors) (Figs 1 and 2). He reported that he had undergone orthodontic treatment 3 years ago but was not happy with the results. He started seeking treatment again for cosmetic reasons. Consultation with multiple cosmetic dentists was sought, and they suggested treating these teeth with veneers or ceramic crowns. The patient had no contributory medical history and reported high physical activity. The patient had an initial consult with a cosmetic dentist who requested an orthodontic consultation. After reviewing and discussing the patient's chief complaint, expectations, clinical and radiographic examinations, multiple options were then presented to the patient. The first option was IPR to reshape the anterior teeth coupled with full comprehensive orthodontic treatment, bleaching, and minor restorative work. The second option was bleaching and veneers or full porcelain crowns. After the discussion with the cosmetic dentist and the patient, he chose the first option. During the first visit, the patient's periodontal condition was recorded and a periodontal examination was carried out. Full orthodontic records of the patient, including radiographs, pictures, and dental impressions were taken.

\section{Treatment Process}

Informed consent was obtained from the patient before starting the treatment plan. The treatment proceeded according to several steps. Study cast measurements, a diagnostic set-up, and calibrated radiographic images

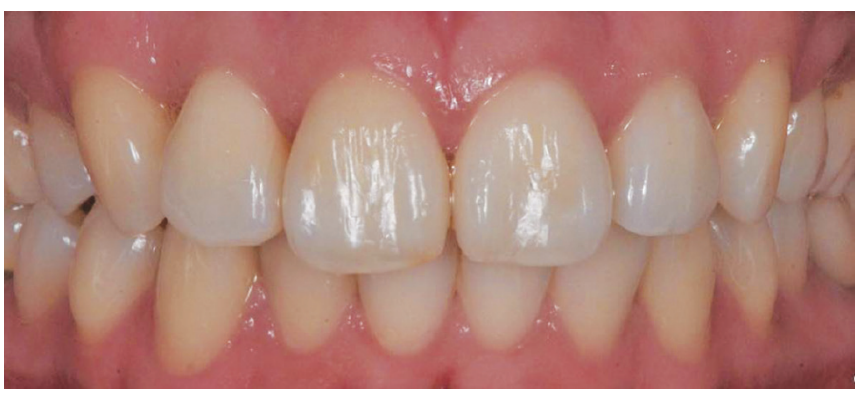

Fig. 1: Retracted frontal view of the patient before treatment

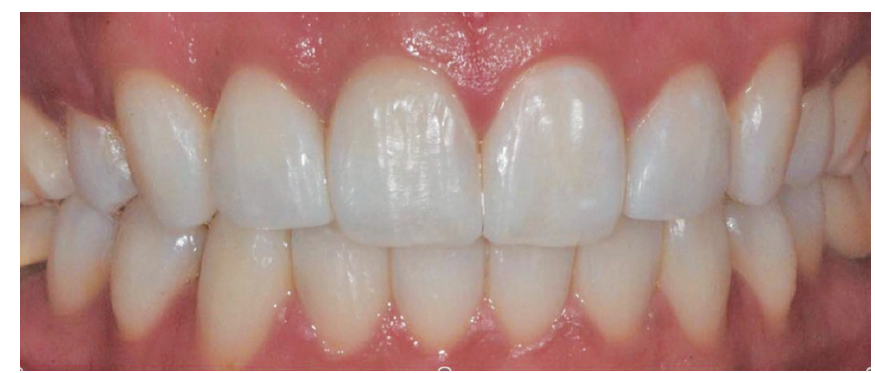

Fig. 3: Retracted frontal view after the treatment were performed. The upper and lower teeth were bonded with a $0.0223 \mathrm{M}$ bracket (Roth prescription). Teeth in both maxillary and mandibular arches were leveled and aligned for 3 months. A steel wire of 0.020 to 0.030 " thickness was placed gingival to the contact point. The IPR for the upper and lower anterior teeth (3-3) was performed using the safe-tipped bur and IPR gauge set from size 0.25 to 0.5 to reshape and give the teeth a sharper masculine look. For finishing and polishing of enamel surfaces, the interproximal corners were rounded with a cone-shaped triangular diamond and white stone burs. Fine sand and cuttle discs (Sof-lex disks, 3M ESPE Dental Products, St. Paul, Minnesota, USA) and finishing diamonds were used to remove surface roughness. To improve the remineralization process of the proximal surfaces, low concentrations of calcium fluoride solution was prescribed for 5- and 10-hour periods. Teeth were retracted to close the spaces using a round wire to decrease the proclination of the anterior teeth and close the spaces. The radiograph indicated that the IPR was still within enamel surface. After closing the spaces, finishing, and detailing, the final teeth positions was carried out for a few months. The case took 7 months to complete the orthodontic treatment. Scaling and root planing were performed, then a lingual fixed retainer was bonded to both upper and lower anterior teeth on the day of debonding. Ten days later, the patient received two cycles of professional in-office bleach for 20 minutes per cycle. In addition, the patient was given a vial of home bleach ( $35 \%$ hydrogen peroxide) to use 4 hours daily for 10 days to obtain a more stable color with less teeth sensitivity (Figs 3 and 4).

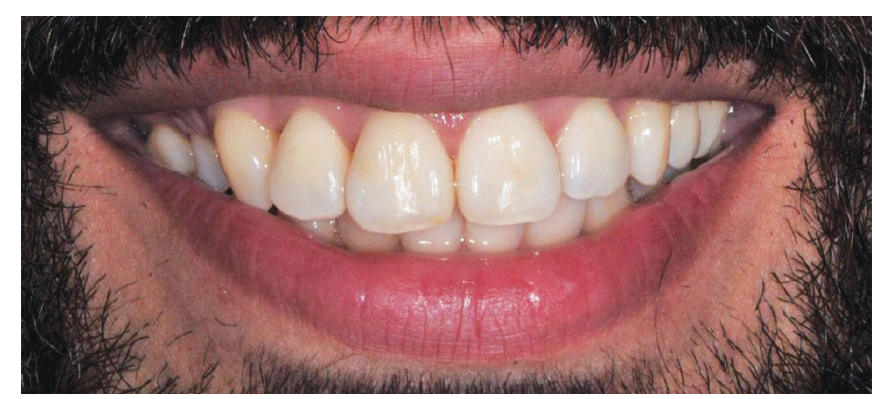

Fig. 2: Smile capture before treatment

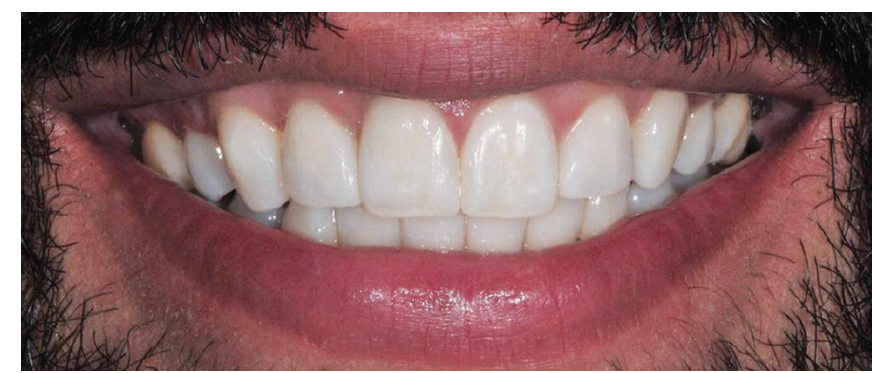

Fig. 4: Smile capture after treatment 


\section{DISCUSSION}

In this case, the patient's chief complaint was resolved with a minimally invasive procedure. The treatment objectives were achieved. The patient finished in Angle's class I molar and canine relationships with optimal overjet and overbite. Interproximal reduction was performed to reshape and contour the upper and lower incisors, which provided adequate space to reduce the incisors' proclination.

Veneers were offered as an alternative option at the initial consultation. The patient opted for the more conservative treatment. Veneers have the advantage of color control. Patients can have their preferred shade and more stable color compared with natural teeth. On average, veneers last for 10 to 15 years, while the orthodontic treatment with IPR treatment option can last for a lifetime. Therefore, the cost for orthodontics with IPR is much less over the long term as veneers require maintenance and replacement after a period of time.,

Orthodontic treatment with interproximal enamel reduction is an alternative, conservative treatment when compared with treatment with veneers. Surfaces of teeth that have undergone interproximal enamel reduction during orthodontic treatment are not more prone to dental caries than untouched surfaces in the same or other patients. It is not yet clear which is the best technique to perform the stripping and IPR. Dental caries incidence was compared between air rotary strippingtreated and unaltered surfaces within the same subjects with no statistically significant differences between the groups. ${ }^{9}$ The incidence in interproximal caries in anterior and posterior teeth with enamel reduction was compared in reproximated and unground surfaces in the same patients with no significant difference to report at 0.05 . Generally, it is recommended to remove between 0.2 and $0.5 \mathrm{~mm}$ per side of tooth enamel according to the need. This is a limitation for IPR in cases that have big discrepancy in the shape of the teeth or in patients with small teeth. Some studies have shown that removal of the whole enamel layer will not induce any side effects. They suggest that the reduction should be performed according to the enamel thickness, fillings, and/or crowns. ${ }^{1,2,4-6}$
Interproximal reduction most of the time in orthodontics is thought of as a treatment modality for creating or gaining space. The IPR can change the shape, dental appearance, and improve gingival contour of teeth. ${ }^{10}$ Also it can improve retention and treatment stability after orthodontics. ${ }^{3,4}$

\section{CONCLUSION}

Interproximal reduction is a valid treatment option that an orthodontist can offer a patient. Cosmetic options for patients should always include IPR, reshaping, and teeth contouring. The orthodontist should not worry about creating an environment that is more susceptible to caries if IPR is chosen since there is no evidence that carefully planned IPR causes tooth decay, sensitivity, or damage to the surrounding tissues.

\section{REFERENCES}

1. Lapenaite E, Lopatiene K. Interproximal enamel reduction as a part of orthodontic treatment. Stomatologija 2014;16(1):19-24.

2. Livas DC. Enamel reduction techniques in orthodontics: a literature review. Open Dent J 2013 Oct;7(1):146-151.

3. Sharma AA, Park JH. Esthetic considerations in interdental papilla: remediation and regeneration. J Esthet Restor Dent 2010 Feb;22(1):18-28.

4. Danesh G, Hellak A, Lippold C, Ziebura T, Schafer E. Enamel surfaces following interproximal reduction with different methods. Angle Orthod 2007 Nov;77(6):1004-1010.

5. Zachrisson BU, Nyøygaard L, Mobarak K. Dental health assessed more than 10 years after interproximal enamel reduction of mandibular anterior teeth. Am J Orthod Dentofacial Orthop 2007 Feb;131(2):162-169.

6. Zachrisson BU, Minster L, Øgaard B, Birkhed D. Dental health assessed after interproximal enamel reduction: caries risk in posterior teeth. Am J Orthod Dentofacial Orthop 2011 Jan;139(1):90-98.

7. Kihn PW, Barnes DM. The clinical longevity of porcelain veneers: a 48-month clinical evaluation. J Am Dent Assoc 1998 Jun;129(6):747-752.

8. Peumans M, De Munck J, Fieuws S, Lambrechts P, Vanherle G, Van Meerbeek B. A prospective ten-year clinical trial of porcelain veneers. J Adhes Dent 2004 Spring;6(1):65-76.

9. Jarjoura K, Gagnon G, Nieberg L. Caries risk after interproximal enamel reduction. Am J Orthod Dentofacial Orthop 2006 Jul;130(1):26-30.

10. Wilcko W, Wilcko M, Bouquot J, Ferguson D. Rapid orthodontics with alveolar reshaping: two case reports of decrowding. Int J Periodontics Restorative Dent 2001 Feb;21(1):9-19. 